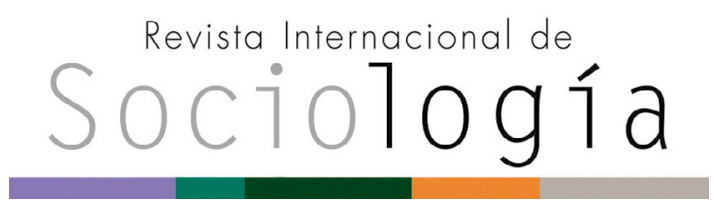

Revista Internacional de Sociología RIS

vol. 79 (1), e181, enero-marzo, 2021, ISSN-L:0034-9712 https://doi.org/10.3989/ris.2021.79.1.19.181

\section{EL NUEVO CENSO DE 2021 EN ESPAÑA: UN DEBATE METODOLÓGICO, EPISTEMOLÓGICO Y POLÍTICO PENDIENTE}

\author{
Andreu Domingo I Valls \\ Centre d'Estudis Demogràfics (CED) \\ Universitat Autònoma de Barcelona (UAB). \\ andreu.domingo@gmail.com \\ ORCID iD: 0000-0003-3270-1939
}

\section{Xiana Bueno}

Centre d'Estudis Demogràfics (CED).

xbueno@ced.uab.es

ORCID iD: 0000-0001-6587-3521.

\author{
Rocío TREVIÑo MARURI \\ Centre d'Estudis Demogràfics (CED). \\ rtrevino@ced.uab.es \\ ORCID iD: 0000-0002-5420-5728
}

Cómo citar este artículo / Citation: Andreu Domingo i Valls, Xiana Bueno y Rocío Treviño Maruri. 2021. "EI nuevo censo de 2021 en España: un debate metodológi$\mathrm{co}$, epistemológico y político pendiente". Revista Internacional de Sociología 79(1):e181. https://doi.org/10.3989/ ris.2021.79.1.19.xxx

\section{Resumen}

En el ejercicio censal de 2021, el censo de población tradicional en España será sustituido por uno basado en enlaces de registros administrativos, complementado con el uso de big data y una encuesta ad hoc. Con el ánimo de suscitar el debate en torno a los desafíos de este cambio metodológico en el ámbito científico, se ha llevado a cabo una investigación cualitativa basada en 30 entrevistas en profundidad, realizadas a productores de estadísticas (Instituto Nacional de Estadística e institutos autonómicos) y a usuarios de tres disciplinas académicas (Demografía, Sociología y Geografía). Las diferentes opiniones, reflexiones, críticas y sugerencias se han ordenado en tres apartados: 1) metodológico; 2) epistemológico; y 3) político, el último referente a la inclusión del big data generado por el sector privado. En las conclusiones se recogen reflexiones sobre el proceso de producción y evaluación del censo, así como sobre los retos que plantea el nuevo método de construcción del censo de población a los investigadores.

\section{Palabras clave}

Demografía, censo, España, estadística, metodología.

\section{THE NEW 2021 CENSUS \\ IN SPAIN: A PENDING \\ METHODOLOGICAL, EPISTEMOLOGICAL AND POLITICAL DEBATE}

Copyright: () 2021 CSIC. Este es un artículo de acceso abierto distribuido bajo los términos de la licencia de uso y distribución Creative Commons Reconocimiento 4.0 Internacional (CC BY 4.0).

\section{Abstract}

For the 2021 census round, the traditional population census in Spain will be replaced by one based on links between population records and administrative registered data, coupled with big data and an ad hoc survey. In order to raise the debate in this regard, 30 in-depth interviews have been conducted with producers -National Statistic Institute and regional Institutes-, and users of census data from three academic disciplines - Sociology, Demography and Geography. The opinions, reflections, criticisms, and suggestions obtained have been organized in three different sections: methodological, epistemological and political, the latter referring to the inclusion of the big data generated by private companies. The conclusions include recommendations on the production process and evaluation of the census, as well as on the requirements for researchers in front of a new method of construction of the population census.

\section{KEYWORDS}

Demography, Census, Spain, Statistics, Methodology 


\section{INTRODUCCIÓN}

El objetivo principal de este texto es promover el debate en torno a la desaparición del censo tradicional en España en 2021 y su substitución por uno nuevo, a partir de enlaces de registros administrativos, una encuesta complementaria ad hoc y el uso de big data.

En el último tercio del siglo XX, la confluencia de las mejoras tecnológicas en la recogida y tratamiento de datos y la creciente oposición de la población al control del Estado, plantearon en algunos países la conveniencia de complementar, primero, y basar, más tarde, los censos de población en los datos procedentes de los registros continuos de población y otros registros administrativos, abandonando las operaciones de campo exhaustivas de recuento de la población y de sus características (Thygesen 2015; Rendfern 1989), siendo pioneros de esa renovación metodológica los países escandinavos. La emergencia del big data y la minería de datos en el siglo XXI aceleraron el proceso en otros países. Como sostiene Louise Amoore (2013), el cambio en el sistema de recuento no solo transforma la medida, sino la forma que tenemos de ver el mundo, cómo nos apropiamos de él y cómo lo categorizamos. Es decir, el cambio metodológico introduce un giro epistemológico y político, aunque estos últimos hayan pasado frecuentemente a un segundo plano en la discusión. Con la llegada de la crisis económica de 2008, y teniendo en cuenta los crecientes costos de unas operaciones censales cuya cobertura y fiabilidad se pondrían en entredicho, aumentó la presión para replantear dichas operaciones.

En España, el Instituto Nacional de Estadística anunció en 2009 su decisión de suprimir el Censo de Población tradicional en el horizonte del año 2021 (Argüeso Jiménez 2014; 2019), habiendo sido el de 2011 un paso intermedio, "ni tradicional, ni del futuro". Con ello se seguían las recomendaciones de la oficina estadística de la Unión Europea (Eurostat) y de la Comisión Económica de la Naciones Unidas para Europa (UNECE) en el ámbito de las conferencias de estadística de mediados de la década del 2000 sobre nuevas metodologías censales (UNECE 2007). Lo que significaría revolucionar la estadística pública española en lo que se refiere no solo al censo, sino a la concepción global de las estadísticas de población, dándose una particular relevancia a los cruces entre estadísticas de población y registros administrativos y explorando las posibles aportaciones del big data. Con todo, esa decisión de gran calado apenas se ha acompañado de un debate en profundidad sobre su alcance y significado, salvo alguna excepción (Ramiro Fariñas 2019).

Es por ello que creemos necesario promover un debate más amplio a partir de la voz de los produc- tores y usuarios de los datos censales, examinando los límites y oportunidades de tipo metodológico, así como las transformaciones epistemológicas que la aplicación de una nueva metodología puede significar desde diversas disciplinas (principalmente la sociología, la demografía y la geografía) y la conexión que tienen con las mutaciones políticas que afectan a la gobernabilidad, en especial, al espacio de información estadística como posibilidad de espacio público (Desrosières 2004) y a la alteración de la división entre lo público y lo privado en la producción y consumo de estadísticas.

Para ello, entrevistamos a productores y usuarios de datos censales y estadísticas de población, a los que queremos agradecer su generosidad y disposición. Personas a las que, pese a mantener en el anonimato en las citas concretas, identificamos por la relevancia profesional e institucional que representan.

\section{FuenteS Y METOdOLOGÍA}

Este trabajo cualitativo ha analizado 30 entrevistas en profundidad realizadas por los propios autores entre mayo y diciembre de 2019 a informantes cualificados. El universo se compone de dos submuestras: usuarios y productores. La primera consta de 20 entrevistas a expertos académicos: demógrafos (7), sociólogos (7) y geógrafos (6) de trece instituciones repartidas en seis Comunidades Autónomas (Cataluña, Comunidad de Madrid, Andalucía, Castilla y León, Galicia y Aragón). Las disciplinas consideradas se nutren, en gran medida, de datos censales para la investigación. La segunda submuestra consiste en 10 entrevistas a productores estadísticos, tanto del Instituto Nacional de Estadística (INE) como de algunas de las CC. AA. con un instituto de estadística propio -Andalucía (IECA), Cataluña (IDESCAT), Galicia (IGE) y País Vasco (EUSTAT)-.

La entrevista constaba de cuatro bloques temáticos: I) Contexto, II) Ventajas, III) Desventajas y IV) Prospectiva. Se analizaron siguiendo un método inductivo y, a continuación, los resultados fueron puestos en común hasta alcanzar un consenso sobre los principales ejes discursivos.

\section{¿UNA CUESTIÓN DE MÉTODO?}

Las ventajas e inconvenientes, la innovación propiamente dicha y cómo se está llevando el proceso de cambio son las tres cuestiones que más preocupan en el ámbito metodológico. Aunque la mayoría de las personas entrevistadas ven el viraje metodológico como "un camino de no retorno", muchos de los usuarios opinan que el giro se ha visto forzado por presiones tanto institucionales (de la Oficina de Estadística Europea, Eurostat) como de financiación (elevado coste del censo tradicional), e incitado, si cabe, por la co- 


\begin{tabular}{|c|c|c|c|c|}
\hline TIPO & DISCIPLINA & NOMBRE & INSTITUCIÓN & CCAA \\
\hline \multirow{20}{*}{ Usuario/a } & \multirow{7}{*}{ Demografía } & Albert Esteve & \multirow{4}{*}{ CED } & \multirow{5}{*}{ Cataluña } \\
\hline & & Montserrat Solsona & & \\
\hline & & Pau Miret Gamundi & & \\
\hline & & Jeroen Spijker & & \\
\hline & & Clara Cortina & U. Pompeu Fabra & \\
\hline & & Diego Ramiro Fariñas & \multirow{2}{*}{ CSIC } & \multirow{2}{*}{ C. de Madrid } \\
\hline & & Teresa Castro Martín & & \\
\hline & \multirow{7}{*}{ Sociología } & Verónica de Miguel & U. de Málaga & Andalucía \\
\hline & & Luis Alberto del Rey & \multirow{2}{*}{ U. de Salamanca } & \multirow{2}{*}{ Castilla y León } \\
\hline & & Mikolaj Stanek & & \\
\hline & & Jesús Leal Maldonado & U. Complutense de Madrid & $C$ de Madrid \\
\hline & & Miguel Requena & UNED & c. de iviauma \\
\hline & & Antonio Izquierdo Escribano & \multirow{2}{*}{ U. de A Coruña } & \multirow{2}{*}{ Galicia } \\
\hline & & Antía Pérez Caramés & & \\
\hline & \multirow{6}{*}{ Geografía } & Aaron Cohen & U. De Granada & \multirow{2}{*}{ Andalucía } \\
\hline & & Jose Maria Feria Toribio & U. Pablo de Olavide & \\
\hline & & Angel Pueyo & U. de Zaragoza & Aragón \\
\hline & & Isabel Pujadas Rúbies & U. de Barcelona & \\
\hline & & Joaquin Recaño Valverde & CED & Cataluña \\
\hline & & Albert Sabater Coll & U. De Girona & \\
\hline \multirow{10}{*}{ Productor/a } & & Fran Viciana & \multirow{2}{*}{ IECA } & \multirow{2}{*}{ Andalucía } \\
\hline & & Juan Del Ojo & & \\
\hline & & Frederic Udina i Abelló & IDESCAT & Cataluña \\
\hline & & Antonio Argüeso & INE & C. de Madrid \\
\hline & & Isabel del Río & \multirow{3}{*}{ IGE } & \multirow{3}{*}{ Galicia } \\
\hline & & Solmary Silveira & & \\
\hline & & Esther López & & \\
\hline & & Enrique Morán & \multirow{2}{*}{ EUSTAT } & \multirow{2}{*}{ País Vasco } \\
\hline & & Nerea Linaza & & \\
\hline & & Ignacio Duque Rodríguez & Estadístico & Agencia Tributaria \\
\hline
\end{tabular}

yuntura económica de la gran recesión. No obstante, si bien los usuarios suelen ver en la crisis económica un detonante de dicha transición, los productores tratan de disociar ambos eventos, convencidos de que se trata de un desarrollo ineludible ligado al progreso.

\section{Innovación y controversia}

"Toda innovación genera controversia", nos advierte una de las entrevistadas. Mientras que los estadísticos se ven a sí mismos como impulsores de la innovación y perciben una actitud melancólica de los investigadores hacia el censo, los usuarios desconfían de las promesas entusiastas de los primeros, parapetados en el escudo de la modernidad. ¿Realmente serán capaces de replicar toda la información recogida por el censo? Esa, y no otra, es la controversia centrada aparentemente en el método.

La mayoría de productores señalan que los académicos siguen anclados en aquello de "todo tiempo pasado fue mejor". En contraste, buena parte de los académicos aceptan la mudanza, incluso la ansían, aunque no sin cierto escepticismo y, circunstancialmente, con cierta nostalgia: "la de quién tiene que despedirse de un viejo conocido". Entre estos últimos, apreciamos un denominador común que tiene que ver con el factor generacional, al que además apela alguna de las entrevistadas como la razón que explica "su dificultad para compartir el optimismo respecto al cambio censal". Se ha calificado el censo como "la piedra angular" o "el soberano de las estadísticas". Muchos se preguntan si el futuro censo será capaz de cumplir los objetivos de universalidad y exhaustividad. Inquieta no saber si la substitución no solo permitirá contar fehacientemente a la población, sino también si podrá reconstruir la información que ofrecía el censo tradicional. Dar continuidad a las series históricas es una de las preocupaciones más recurrentes. A pesar de ello, también hay quién ve en el nuevo modelo una oportunidad para la inclusión de información novedosa. "El censo dejará 
de ser una fotografía, tiene que ser más una mirada hacia el pasado", afirma uno de los entrevistados en referencia a la inclusión de registros de carácter longitudinal.

La calidad de los datos que van a enlazar es lo que despierta la mayor desconfianza. Dudas que apuntan a los cuatro grandes componentes del nuevo censo: el padrón, los registros administrativos, el big data y la encuesta complementaria. Al padrón, que constituirá el pilar del nuevo censo, se le atribuyen numerosos problemas de registro sobradamente conocidos (entre otros, los falsos empadronamientos, la desactualización de los datos, el registro concerniente a la población extranjera). De la calidad de los registros administrativos se sabe que se encuentran a merced de la relación que el ciudadano pueda tener con la Administración Pública, por lo que pueden reflejar un "retraso" en la veracidad de los datos. Sobre el big data, la objeción preponderante apunta al propósito original con el que fueron recogidos y cómo éste pueda compatibilizarse con el de la estadística pública. Mientras que a la encuesta complementaria se le presupone, de partida, insuficiente para solventar cuatro aspectos que se señalan como carencias de la nueva metodología censal en relación al censo tradicional. Primero, el detalle territorial y la movilidad cotidiana, que preocupan principalmente a los geógrafos. Segundo, la información relacionada con la estructura del hogar, vivienda, relaciones de parentesco, ocupación o nivel socioeconómico, que reportan sociólogos y demógrafos. Tercero, la población invisibilizada por no poder ser observada, entre quienes se menciona a colectivos vulnerables (migrantes o personas en riesgo de exclusión social), pero también a poblaciones transitorias (transeúntes, nómadas). Y cuarto, la población no encontrada, como jubilados o estudiantes. Se tiene la percepción de que éstos importan más a los científicos sociales que al INE, cuya misión es la de dar una visión de conjunto. "Son poblaciones que, si no las podemos estudiar bien, difícilmente las podremos mejorar", alerta una investigadora. En cualquier caso, existe un consenso generalizado en cuanto a la falta de concreción del INE -incluso en documentos oficiales como el anteproyecto del censo de 2021- sobre el producto final y su contenido, y es dicha inconcreción la que, en parte, alienta la cautela del usuario y genera dudas. "A día de hoy hay muchos puntos oscuros" comenta un entrevistado. Si bien, los más optimistas son también más pacientes y confían en el potencial del nuevo modelo: "Hasta que no se haya producido el producto censal de 2021 y el INE no tenga bien engrasada la maquinaria del enlace de registros, posiblemente habrá problemas", nos comenta un usuario y añade: "es una operación de muchísima envergadura en la que hay que atar muchísimos cabos".

\section{La revolución de los datos: el mundo que viene}

Los productores se ven a sí mismos como abanderados del futuro, o en sus propias palabras: "En ese mundo hay que entrar, el mundo está cambiado y tenemos que cambiar con él". A diferencia de los académicos, los productores no ven en el censo tradicional tantas bonanzas y se refuerzan en la idea de que era un producto anticuado, casi anacrónico y que el avance tecnológico ha contribuido a una revolución en la forma de generar datos. La combinación del registro de población continuo (el Padrón) y su enlace con registros de naturaleza administrativa, encuestas y big data se presentan como la fórmula mágica del nuevo modo de conteo de población. "Más vale que todos nos vayamos acostumbrando a pensar en estos datos y a utilizarlos", afirma uno de los usuarios. Además, el abaratamiento de los costos y la simplificación logística, por una parte; y el aumento de la periodicidad, evitando esperas decenales, por otra, son las dos grandes ventajas por antonomasia que aportará el cambio metodológico y que más refieren productores y usuarios. La revolución de los datos hace posible el aprovechamiento del numeroso repertorio de registros administrativos existentes, aportando valor añadido. Se prevé, también, que la información sea de mayor calidad al ser contrastada en varios registros y se valora el volver a tenerla para toda la población, a diferencia del censo de 2011. A juicio de un productor, con todo, esta calidad dependerá de la mejora de los repertorios unificados de direcciones locales, callejeros, viviendas, identificadores, coordinación con las instituciones productoras de registros... en definitiva, de la mejora de la infraestructura estadística, que lamenta no esté dando sus frutos.

Desde la perspectiva del uso, la revolución de los datos representa también una oportunidad de innovación metodológica. El enlace de registros se considera una ventana abierta a los tan ansiados datos longitudinales e instan al INE a recoger información biográfica. No obstante, hay quién se queja de que se plantee el proceso simplemente como de sustitución, cuando debería tratarse como un proceso de complementariedad gracias a la adición de nueva información. Una de las sugerencias reiteradas es la de usar la encuesta complementaria para la recogida de datos retrospectivos, emulando la Encuesta Sociodemográfica de 1991. Esta manera de entender la producción abriría una vía a originales diseños de investigación antes impensables, a través del enlace de ficheros ad hoc. Se espera, asimismo, que el nuevo censo conlleve mayor interdisciplinariedad en la investigación al generar sinergias entre, por ejemplo, científicos sociales y analistas de datos masivos (matemáticos, ingenieros informáticos); además de generar una innovadora 'cultura registral', facilitan- 
do progresivamente la explotación de registros hasta ahora inéditos.

\section{¿Estaba preparado el INE?: asomarse al abismo}

Algunos entrevistados temen que "de la realidad estadística a la realidad de la calle pueda haber un abismo". No se refieren al cambio, ponen en duda el 'tempo', la precipitación. "Dar pasos tan importantes y poco meditados metodológicamente irá en detrimento de los datos", sentencia un investigador. Los trabajos de depuración, armonización y enlace de registros se prevén arduos y un tratamiento deficiente de sus impurezas podría desvirtuar la calidad. Existe un cierto recelo sobre la transparencia metodológica. Si bien, también es cierto que no toda la comunidad científica desea involucrarse en dicho debate metodológico. En este sentido, un usuario opina: "muchos [investigadores] prefieren que les den un producto hecho sin importarles cómo está hecho". Otros lamentan que no haya habido un proceso participativo en el que el INE tomara en consideración las aportaciones de técnicos, científicos sociales, la administración local y el público en general. "Más vale un debate tarde que nunca", comenta esperanzada una de las entrevistadas.

Los productores tienen la convicción de que tanto el padrón continuo como los registros administrativos están suficientemente maduros para afrontar la empresa. Además, enfatizan la gran ventaja de que España cuente con un registro municipal de población que servirá de 'esqueleto' al nuevo censo. Su opinión respecto al modelo tradicional es tajante: es inviable. Lo es tanto en términos económicos como humanos, pero, al tiempo, trasladan parte de la responsabilidad a la población, que "ya no quiere responder cuestionarios". Los productores recalcan el cansancio del ciudadano para proporcionar información que ya existe en los registros administrativos (Bechtold 2012). Es destacable que diversos estadísticos enumeran ahora las muchas debilidades que acarreaban los censos tradicionales por las dificultades operacionales que implicaban. "El último gran censo fue el de 1991". Pero también uno de ellos señala que la "anomalía" del censo de 2011, de no ser exhaustivo, penalizará la calidad del próximo "al faltar una referencia a la que acudir para aquellos colectivos de los que se va a perder información con el cruce de los registros administrativos".

Quienes dependen de las estadísticas, por su parte, mencionan que hubieran sido necesarios 'ensayos' previos con el enlace de registros. Existen precedentes sobre las que nos han hablado los estadísticos de Andalucía, País Vasco o Galicia. Dichos institutos autonómicos llevan tiempo trabajando en la construcción de bases de datos enlazadas. Sin em- bargo, el INE no parece haber sido muy proclive al intercambio de estas experiencias con los institutos estadísticos. Algunos usuarios deploran que no se haga una "economía de escala", no solo con institutos de estadística autonómicos, sino también con los ayuntamientos. "Se debe situar a la administración local en un primer plano gracias a la transformación digital", sugiere uno de ellos.

La falta de confianza en la idoneidad del momento lleva a numerosos investigadores a criticar la falta de cuestionamiento respecto al cambio, la falta de debate académico, así como la inexistencia de una reflexión sobre lo que supone el uso de registros administrativos. Se reclama una evaluación de la calidad de los registros o un informe exhaustivo de las incidencias acaecidas. Preocupa el "cambiar sin evaluar", y se requiere una auditoría de las decisiones metodológicas tomadas por el INE en este proceso de transformación que evalúe los riesgos, ventajas, e inconvenientes, ya que se están enlazando fuentes creadas con fines muy distintos. No pocos han mencionado la necesidad de que el INE informe si los datos ofrecidos son reales o estimados y, en su caso, cómo lo han sido. Asimismo, a diversos investigadores les desasosiega cómo se van a operacionalizar el uso y análisis de datos privados, es decir, la comercialización del big data. Al respecto, el propio INE dio a conocer en diferentes declaraciones públicas que, si bien inicialmente esperó un acceso libre de obstáculos a los datos de telefonía móvil para el análisis de la movilidad cotidiana, finalmente convinieron en la necesidad de obtenerlos previo pago, dada la incapacidad del propio corpus estadístico de armonizar los datos brutos para su ulterior análisis. Dicha transacción fue objeto de polémica y estuvo alimentada en los medios de comunicación por el miedo al control e invasión de la privacidad ciudadanía por parte del INE. Controversias similares se dieron en otros contextos europeos (Dugmore et al. 2011).

La difusión de los datos y su accesibilidad constituyen una pieza clave y anexa a la metodología. Mientras se promete un flujo continuo (anual) de datos a partir de 2021, algunos entrevistados —los más visionarios - fantasean con la posibilidad de solicitar cruces de registros ad hoc que satisfagan sus inquietudes científicas. En general, el investigador tiene la expectativa de que el INE mantenga la política de accesibilidad que ha ofrecido hasta ahora, facilitando una muestra anonimizada de microdatos. Política que es valorada muy positivamente. De todos modos, una cuestión es el producto censal -como muestra gratuita- y otra es el cruce de registros bajo petición. En referencia a esto último, existen tres grandes interrogantes. El primero es el coste. En la actualidad, ciertos datos con mayor desagregación tienen un coste relativamente asumible. El usuario prevé que el cruce de datos ad hoc implicará un coste, quizás no al alcance de todos. Esto iría, a 
priori, en detrimento de la democratización de los datos, entendidos como un bien público a disposición del ciudadano. El segundo aspecto tiene que ver con la paradoja entre el aumento de la riqueza de datos y el incremento proporcional de las restricciones de acceso en nombre de la confidencialidad. El tercer aspecto es el de la capacidad del propio investigador de manejar datos a partir de un cierto volumen. $Y$ es que el cuestionamiento metodológico no solo afecta al INE: algunos académicos también se preguntan si la comunidad científica está lista para el cambio. Se destaca la escasa masa crítica que existe a nivel científico y que se explica por una falta general de formación reglada en el análisis de datos y, especialmente, de datos masivos.

\section{IMPLICACIONES EPISTEMOLÓGICAS}

\section{Construcción, significado y naturaleza de los datos}

Para gran parte de los productores, la información de registros y de un censo tradicional son intercambiables: "se perderán unas cosas, pero se ganarán otras", dicen, sin considerar la diferente naturaleza de los mismos. No obstante, todos distinguen entre la naturaleza del big data y la de los registros administrativos. A los primeros se les considera "asistemáticos, muy sesgados, con ruido, de mayor exigencia tecnológica en su tratamiento, sin continuidad, no demandados sino captados, pagados y con implicaciones éticas de mayor hondura". Pero en la recolección de datos administrativos también se replantea un cambio de paradigma que, en principio, podría dibujarse más inductivo que deductivo: "tenemos que cambiar el chip de que queremos aquellas variables, pues a lo mejor no", el ámbito de elección entre producción y uso se achica en función de lo que la "sociedad provea".

Si la recogida de información en un censo tradicional se realizaba para un conjunto limitado de propósitos, el nuevo censo parece dibujar un modelo de producción estadística en la que la relevancia del dato será medida en relación con aquello que quede registrado. El espacio de una aproximación deductiva, que parta de una observación enmarcada teóricamente, no queda eliminado, pero sí puede reducirse. Se es consciente de esa alteración: "en el sistema de registros, lo que está en el registro existe y lo que no está no existe". En otros casos, se resalta el carácter polisémico del dato del registro, con la capacidad de adquirir nuevo significado dependiendo de la vinculación a otros registros. La construcción de la herramienta de conocimiento pasa por la conceptualización del enlace de datos registrales.

Para los productores y para algunos usuarios, el dato será "más preciso, objetivo y contrastable", pu- diéndose cotejar además con diferentes fuentes administrativas. Para otros, el dato refleja el fin para el que fue recogido y para dar significado al mismo se necesitará información adicional que lo contextualice: cómo fue producido, para qué, por quién, cuándo y de quién fue recogido, "más que los microdatos, me preocupan mucho los metadatos"; "¿qué tiene montado ahora [el INE] para intentar juntar registros que vienen de distintas entidades que no son el propio INE?". La producción de información puede conllevar la alienación del investigador de su objeto de estudio, "con qué propósito está hecho cada uno, cuál es nuestra pregunta de investigación y la finalidad". Se percibe en las respuestas la complejidad de dar significado a los datos surgidos de los registros. Para los productores puede suponer un reto: "si fuéramos un poco más letrados, en el sentido de abogacía, deberíamos incluso conocer más la normativa que está detrás del dato de registro"; para los usuarios, desconcierto: "antes teníamos una fotografía y puede que ahora tengamos un puzzle".

La transparencia en la codificación de la realidad resulta ahora menos nítida porque entran en juego algoritmos estadísticos que el investigador desconoce: "cuando tienes información que te ofrece información distinta tienes que jugar... digamos a un algoritmo de fuentes ganadoras". Sin embargo, el problema que se plantea es la construcción del "algoritmo ganador". Como apuntan muchos entrevistados, existe muy poca reflexión teórica sobre la incertidumbre que rodea la validez de los datos de registro y su enlace (Holt 2007). Cómo conceptualizar y medir la precisión estadística de los datos de registros aplicando conceptos estadísticos rigurosos como los de sesgo, varianza, eficiencia o consistencia todavía está en sus primeras fases de investigación (Zhang 2012). Los sistemas administrativos incorporan problemas de cobertura, de umbral y de definición conceptual de las variables que son específicos (Holt 2007). Esta preocupación se repite en las entrevistas, como eco de algunos de los debates internacionales, entrando en juego el balance entre la inmediatez y la pérdida de precisión conceptual y de cobertura.

\section{Población, territorio e historia}

Las dudas de los usuarios sobre el próximo censo se articulan en función de la disciplina de procedencia en tres dimensiones: población, preferentemente desde el campo de la sociología y demografía; territorio, desde la geografía; perspectiva longitudinal del enlace de registros, resaltada desde la demografía y la demografía histórica.

Las desconfianzas sobre una correcta contabilización de la población por la irresuelta problemática de la medición de la residencia en el padrón inquieta a 
los demógrafos, ya que los datos censales constituían "la regla de oro", sus denominadores. Demógrafos y sociólogos nos previenen de los puntos ciegos de representatividad y de los sesgos que pueden tener los registros, no dando cuenta de aquellas poblaciones que participan en el mundo social sin dejar huella. El censo permitía acceder a toda la población y fue un instrumento fundamental en los inicios del Estado moderno para la creación de conocimiento, nuevos objetos de intervención y para la constitución de formas de Estado no autoritarias (Curtis 2012), y gran parte de las opiniones de los entrevistados expresan las implicaciones que el cambio de metodología censal tendría en cada una de estas dimensiones. Manifiestan la necesidad de analizar el lado derecho y, sobre todo, el izquierdo de la curva de Gauss, reclamando el conocimiento de la variabilidad de las poblaciones como herramienta de progreso social y reconociendo el valor de los datos para la acción de gobierno, especialmente, a nivel local. Lamentan así la paradoja de que fuese precisamente en uno de los momentos recientes de mayor transformación demográfica del país, con la llegada del boom inmigratorio internacional, cuando el censo de 2011 optase por el uso muestral, dificultando el acceso al conocimiento de estas poblaciones. Los productores alegan, en unos casos, que realmente el censo tradicional tampoco accedía a estas poblaciones; en otros casos, se aboca al investigador a la búsqueda de nuevas aproximaciones cualitativas: "tenéis que buscar enfoques diferentes que seguramente han de tener un componente cualitativo bastante importante", $\mathrm{y}$, a veces, se plantea como un problema a solucionar en el futuro. Un elemento más de contraste entre estadísticos y usuarios es el valor que se da a los comportamientos minoritarios, porque mientras para unos escapan al interés público general regido por la ley de los grandes números, para otros, como indicativos de la emergencia de una mutación social, son trascendentales. Algún productor, que enlaza con las preocupaciones del usuario, señala la poca reflexión habida en "revertir los aspectos negativos de una doble cifra de población en las estadísticas demográficas".

Si en los debates internacionales se destaca el censo de población como la fuente estadística de los geógrafos por antonomasia y, por tanto, la naturaleza manifiestamente espacial de alguna de las fortalezas y debilidades de las nuevas metodologías censales (Skinner 2018), así también es en las voces de la geografía en las que se producen las objeciones más enconadas al abandono del censo tradicional. Las negativas repercusiones que tuvo el censo de 2011 para el análisis de las unidades territoriales pequeñas, es decir, para lo que se conoce como granularidad, definida como la capacidad de producir estimaciones precisas para subgrupos pequeños, fundamentalmente áreas pequeñas, condiciona la opinión de muchos geógrafos. Ven el censo de 2011 como un anteceden- te de lo que será el próximo y de las devastadoras repercusiones que tendrá el estudio de lo social en el espacio. Para productores la georeferenciación soslayará la necesidad de una determinada geografía de recogida de los datosy añadirá la flexibilidad para determinar el ámbito geográfico de estudio, por ejemplo, las poblaciones en riesgo, lo que facilitaría una herramienta de trabajo más polivalente (Skinner 2008). Los productores destacan así las oportunidades que el nuevo modelo brindará a los geógrafos, al poder crear sus propios polígonos de información solo sujetos a los umbrales de confidencialidad. No obstante, algunos de los entrevistados cuestionan la calidad de la georreferenciación, ya que los registros no tienen una fecha fija y existen numerosas contradicciones incluso entre direcciones registradas en una misma administración, advirtiendo que ello redundará en "una cosa borrosa en el tiempo". Otro aspecto que inquieta es cómo se alcanzará el territorio en las variables procedentes de la encuesta y si, en aras de salvaguardar el anonimato, disminuirá la trasparencia en la imputación aumentando "el ruido" e impidiendo el tratamiento individual, no agregado, de los mismos, limitando así el análisis multivariado en escalas territoriales pequeñas.

Mención aparte merece la opinión que se tiene sobre las oportunidades que ofrece el big data, dado que sus atributos tienen en gran parte una referencia espacial. Se ve como oportunidad y como riesgo. Como oportunidad de acceso a nuevas fuentes de datos y de financiación y como riesgo de que la digitalización favorezca la proliferación de gurús de la ciencia de los datos y la banalización de su disciplina. Así se expresa, al respecto, otro de los entrevistados: "incluso ahora estamos viendo que los periodistas (...) ya hacen la labor que hacían antes los geógrafos (...) tienen acceso muchas veces a big data (...) parten de una idea, que yo creo que es una falacia, que con los datos ya pueden establecer cosas y yo creo que analizar datos sin teoría... [es peligroso]". Como plantea Kitchin (2013: 263), la aparición de un nuevo paradigma de la "ciencia basada en datos" desafía las epistemologías establecidas mediante la combinación de enfoques abductivos, inductivos y deductivos.

Además del continuum transversal del tiempo, a los sociólogos y, sobre todo, a los demógrafos les preocupa que los datos censales integren el ángulo longitudinal: "el INE tendría que tener en el censo una actitud un poco más, diríamos, más abierta, e incorporar la información con un orden de prevalencia temporal". En demografía, como en el caso de la cartografía, los fundamentos ontológicos de la disciplina transitan también desde una comprensión descriptiva de cómo son las cosas a una ontogenética de cómo han llegado a ser (Kitchin, Gleeson y Dodge 2013). El seguimiento de grupos poblacionales a través del tiempo define la norma legítima de la actividad científica del paradigma dominante en 
demografía (Event History Analysis), que formula el análisis de los eventos biográficos de las personas en términos de procesos sociales (Courgeau y Lelièvre, 1997). Las expectativas inmediatas, y no tanto de futuro, de lo que supondrá el próximo censo en información longitudinal entre los productores y receptores son muy dispares.

\section{Institucionalización, competencia y docencia}

La institucionalización de la investigación científica, la docencia y la producción estadística también se verían afectadas por el desafío epistemológico que supone el cambio censal. En el ámbito de la institucionalización, algunos plantean la necesidad de crear estructuras interdisciplinares para hacer frente a los retos que supone el nuevo modelo estadístico. También, como en otros países, preocupa que la competencia de disciplinas como ingeniería, informática o física en la interpretación de procesos sociales acabe obviando todo el acervo de conocimiento acumulado de la ciencia social (Kitchin 2013). La potencialidad infinita de producir información estadística y a la carta, a partir de los registros administrativos, exige una organización más eficiente que, en algunos casos, los entrevistados sugieren que se haga a través de estructuras intermedias: "A España le falta una pieza que tienen la mayoría de países (...) no son los INE los que hacen estas encuestas sino unas instituciones intermedias, como date archive, como los consorcios de investigación y los INE están para hacer estadística oficial". Efectivamente, en algunos países, como en Suecia o Países Bajos, se crearon este tipo de infraestructuras para la producción de datos longitudinales (Vikström, Edvinsson y Brändström 2002).

Ligado a lo anterior, se plantea un segundo desafío que tiene que ver con la cultura registral. Los entrevistados se quejan a menudo de que los datos de los registros "son de su padre y de su madre", planteando la necesidad de que los gestores de la información tomen conciencia del gran activo que esos datos tienen para el diseño de políticas públicas. Esa cultura registral no solo debería enfocarse a la protección de la privacidad de los ciudadanos, sino también a la transparencia administrativa y a la responsabilidad democrática: "Para mí una infraestructura de Estado necesaria sería poner un poco de orden en la recolección, armonización y fusión de datos estadísticos".

La escasez histórica de recursos de investigación y de datos ha provocado una tardía institucionalización de las disciplinas sociales en España y una orientación hacia una ciencia poco apoyada en datos, lo que, a su vez, les ha restado competencia en los foros internacionales y, provocando hasta ahora, poca presión a la demanda de datos. Hay voces que enfatizan que el nuevo sistema representa una oportunidad para acercar la producción estadística a los intereses del investigador, pero también supone un reto para la docencia. La producción a la carta, sus costes y su complejidad pueden estar alejando la docencia de la investigación.

\section{Big DATA Y ESTAdíSTICA PÚBlicA: "COMO EL ACEITE Y EL AGUA"}

Las respuestas sobre qué papel puede jugar el big data en la confección de estadísticas sobre población en general y sobre el censo de 2021 en particular, esta vez sin apenas distinción entre usuarios y productores, oscilan entre el entusiasmo por las posibilidades futuras y el escepticismo sobre el alcance de sus aportaciones. Aunque suelen referirse a datos de distinto origen: mientras que los productores piensan más en datos procedentes de compañías de servicios, el usuario lo hace en los de las redes sociales. Lo que se pone en duda es la adecuación de los registros y el big data con la estadística pública, teniendo en cuenta la distinta naturaleza de los primeros, relacionada con el negocio privado, con el consumo y la vigilancia; y, por otro lado, de la estadística pública, que no solo crea el espacio de enumeración y nominación que corresponde ejemplarmente al censo, sino que se debe al bien común. Como resumía uno de los productores: "El big data y la estadística oficial son como el aceite y el agua, dos mundos muy diferentes".

\section{Entusiasmo y escepticismo: "renovarse o morir"}

El entusiasmo se debe a las posibilidades de conocimiento de la población y el territorio gracias a la capacidad de análisis de datos masivos. Se menciona la posibilidad de tener información que ahora escapa al registro censal, permitiendo distinguir entre población vinculada y flotante, gracias a los datos de telefonía móvil, que nos informan sobre la población estacional, también de la movilidad o de la ocupación de la vivienda (a través del consumo energético). Información que, sin duda, resultaría de gran utilidad para la gestión de los servicios públicos y la ordenación territorial. También se apunta que esta nueva forma de medir poblaciones y sus comportamientos, podría abrir un espacio incógnito en campos como la salud, a partir del consumo sanitario y, en general, con los comportamientos demográficos, integrando los análisis de las redes establecidas mediante Facebook o Twitter, por ejemplo: desde la fecundidad hasta las migraciones. Otros creen que sería de utilidad para complementar estadísticas de población sobre las que se sospechan sesgos, como 
acontece con las migraciones deducidas a través del empadronamiento, tanto de extranjeros como de nacionales. En todo caso, esas posibilidades siempre se proyectan en el futuro: una promesa, irreversible, pero aún no una realidad. Se duda sobre el alcance de su aplicación en el censo de 2021. La diferencia radica entre aquellos entusiastas que lo perciben como la puerta a ese porvenir, no solo "el big data es el futuro", como nos afirma uno de ellos, sino que "es el futuro, y son la manera de que nosotros [los estadísticos] tengamos futuro"; y, hasta aquellos otros que creen que puede ser un componente más de ese futuro, pero con limitaciones considerables.

Parte de ese entusiasmo es debido a la novedad, expresada en términos de "momentum" por un usuario. Se prejuicia de unos resultados que expresan la fascinación estética y el número como fetiche, sin dar cuentas sobre de su fiabilidad: "Veo cosas muy... fashion en internet, cosas muy bonitas que se pueden hacer con super mapas y tal, pero me da la impresión que hay mucho humo también". En esa crítica más centrada en lo metodológico, en que se sacrifica la precisión por la actualidad, enunciada en positivo por un productor como: "menos exacto, pero más al día", se destacan cuatro elementos: la dudosa representatividad -("El big data pone números a la cotidianidad, dónde hacemos más fotos, con quién nos relacionamos etc., pero vulnera todos los principios de representatividad en el análisis")-, la dificultad o imposibilidad de establecer relaciones causales -("Para mí, las ciencias sociales de corte cuantitativo están avanzando cada vez más a crear diseños longitudinales que permitan mirar causas y efectos, (...) pero con el big data no puedes")-, la falta de estructuración de los datos -("...yo siempre he trabajado con datos estructurados, el big data, una de las cosas que tiene es la no estructuración de los datos")-, y de la inseguridad respecto a la continuidad de las series. Esta última, no por falta de voluntad de los productores, sino porque la metodología utilizada se vuelve obsoleta con mucha rapidez, cuestionando, por un lado, los resultados anteriores; pero, sobre todo, la interpretación evolutiva de los mismos.

Ese problema metodológico revierte en uno epistemológico que no se circunscribe a la utilización del big data, de modo que la fragmentación de las series estadísticas repercute en una fractura de la posibilidad de progreso en la investigación. En los países escandinavos, se nos advierte, con mejores datos administrativos y longitudinales, pueden formularse preguntas de investigación mejores. Pero, además, la falta de estructuración, debido al carácter caótico de los datos recogidos por el big data lleva a la disolución de la capacidad interpretativa, como previene uno de nuestro entrevistados: "yo creo que analizar datos sin teorías o sin... digamos, algún tipo de estructura previa, nos lleva a la paradoja que muchas veces ha contado Borges en sus cuentos, por ejem- plo, "Funes el memorioso", que es el hecho de que podamos tener toda la información del mundo y no podemos decir nada porque tenemos que decir: ahí está el mundo".

\section{Lo público, lo privado y lo común}

El censo puede ser pensado desde la óptica política como una piedra angular de la gobernabilidad moderna (Lutz 2008). La posibilidad de realizar enlaces de registros administrativos y de la ampliación a la minería de datos y el big data no es únicamente una cuestión metodológica, plantea de forma implícita el papel del Estado en la creación de la estadística pública, sacando a la palestra la redefinición de lo público, lo privado y lo común.

La explicación recurrente del ahorro de presupuesto en la eliminación de la operación censal ensombrece que una parte substantiva de la aceptación de ese cambio se deba también a la colonización de la lógica empresarial sobre lo público, de raíz neoliberal. En los institutos de estadística, la reducción de personal y la maximización de beneficios (que incluye tanto la contención presupuestaria como la comercialización de datos) podrían estar pervirtiendo el sentido originario del servicio público. El origen y objetivo del big data en el sector privado es la obtención de beneficios (optimizando la venta del propio producto al incorporar la información del comportamiento y gustos del cliente, o convirtiendo los datos extraídos de los usuarios en mercancía); y más tarde, el control de la población. Aunque se citan excepcionalmente trabajos de ciencia ciudadana que, utilizando el big data, han conseguido coproducir investigaciones que tienen como única finalidad el bien común, lo habitual es considerar la minería de datos como un elemento asociado a las grandes corporaciones. Como asegura uno de nuestros entrevistados, estadístico: "hay un riesgo de que esos sistemas que son realmente muy poderosos nos intenten sustituir porque, además, esto es un negocio, la información son muchas cosas y es una fuente de poder y una fuente de riqueza, desde mi punto de vista los poderes públicos no deberían permitirlo por muchas cosas, porque nosotros somos representantes realmente de la población, tenemos un mandato que en teoría es el bien común, cosa que no tienen esas grandes empresas". El big data supone, desde esta óptica, una amenaza para la estadística pública: "(...) en ningún caso podemos dejar que el big data acabe con la estadística, es un gran riesgo porque lo estamos viendo", lo que daría cuenta de la erosión del Estado frente a las corporaciones, grandes productoras. Lo que está en entredicho es el gobierno de la población.

$\mathrm{Y}$ aunque el tema del control se plantee de forma excepcional, la opacidad del objetivo y recogida de los datos, de los algoritmos que se aplican desde las empresas trasciende una vez más las consideraciones de carácter puramente metodológico. Eso nos dice un 
usuario: "(...) entonces yo lo que propongo es saber con qué propósito está hecho cada uno [de los algoritmos utilizados], cuál es nuestra pregunta de investigación y la finalidad, pero necesitaremos que estadísticos y matemáticos nos ayuden (...) me gustaría conocer cuáles son esos maravillosos algoritmos que van a gestionar la información...". Eso, por no hablar de la información que las empresas están dispuestas a ceder o vender a las entidades públicas y de la dependencia que, hoy por hoy, tienen tanto productores públicos como usuarios de saberes reclutados por el sector privado. En su versión positiva, se habla de interdisciplinariedad forzada, debido a la incapacidad de una sola disciplina de hacer frente a la sofisticación metodológica del big data, por un lado, y a la capacidad interpretativa, por otro: "los sociólogos o científicos sociales pasaremos de la teoría a la práctica y tendremos que hacer equipos interdisciplinares sí o sí, porque no sabremos cómo manejar gran parte de esos instrumentos". Para alguno de los geógrafos, el desapego por lo territorial, junto con esa dependencia creciente 0 , si se quiere, la falta de inversión en un sector que, por definición, debería contemplar su actividad como apuesta por la gobernabilidad y el bien común y no como gasto, traduce esa renuncia del Estado a seguir conformando un modelo de garantía de lo público. Como se nos advierte: "El problema es quién va a tener el big data, ¿va a ser la Administración Pública para generar su propio big data o va a comprarlo a unos operadores que en un momento determinado cambian los modelos de recogida de datos o que restringen el acceso a los mismos o que desaparecen y te dejan, digamos, desvalido para poder acceder a información?" No solo por la dependencia de productores externos, sino por la ignorancia de los criterios, la dificultad de acceso, la falta de continuidad y el límite de la posibilidad de cruzar datos que caracterizan las series estadísticas producidas sobre la base del consumo. Otro investigador responde: "Los institutos de estadística están obligados a convertirse, ellos también, en productores de big data". Al big data le corresponde, por su propia naturaleza, la atomización de los datos, mientras que la aspiración del Estado es lógicamente su unificación. Hay una aparente contradicción en pensar el big data como ejemplo de "El Big brother" - entendiendo por éste el Estado autoritario-y la situación actual, donde es precisamente la empresa privada, la que amenaza con ejercer el control absoluto. El miedo al "Big brother" habría provocado los primeros movimientos contrarios al censo en los países escandinavos con la agilización del cruce de los registros administrativos como substitución del censo tradicional. En cambio, en la situación actual, el peligro de la vigilancia se expresa en términos de la debilidad del Estado, su supeditación al big data producido por el sector privado, lo que Shoshana Zuboff (2019) ha llamado "El Big other", que caracteriza al capitalismo de la vigilancia como aquel que reclama la experiencia humana como materia prima de prácticas comerciales basadas en la predicción y venta de las mismas.

\section{Conclusiones}

El remplazo del censo tradicional plantea retos metodológicos, tanto a los productores como a los usuarios, retos que arrastran consideraciones epistemológicas de calado y que, sin embargo, están siendo soslayadas. Hay demasiado en juego: el conocimiento sobre la realidad demográfica, la capacidad de gobernar a partir de ese conocimiento y la presencia de la investigación española en el ámbito internacional, que, debido al déficit de participación en las grandes encuestas internacionales y de información longitudinal, pierde competencia. En esos retos desaparece la dicotomía entre productores y usuarios, porque el objetivo es el mismo. Con el próximo censo de 2021 veremos si el cambio significa la apertura a una nueva posibilidad de la estadística pública o si, en el camino, hemos ido perdiendo información y rigor. Si la imagen se hace más y más borrosa, cediendo a la incertidumbre y pasando a las conjeturas o si, por el contrario, conseguimos una más nítida, actualizada y rica, como se nos promete. Pero quizá el INE debería comprometerse con una evaluación pública de los resultados del censo, como culminación del propio proceso censal. Redundando ésta no solo en la transparencia de su actividad, sino en la mejora de lo que inevitablemente se perfila como un proceso continuo. La exigencia se proyecta también sobre el usuario, con la necesidad de profundizar en la interdisciplinariedad, pero también la de responder al esfuerzo estadístico con una mayor reflexión sobre las implicaciones de la producción del dato integradas en la investigación correspondiente y en el aprovechamiento máximo de las oportunidades que efectivamente se brinden.

La substitución del censo tradicional plantea interrogantes que van más allá de la simple bondad y la cobertura de la información obtenida mediante nuevas fuentes y metodologías respecto a la que se obtenía anteriormente. Puede ser pensada como una prueba de la transformación del propio Estado y un campo de redefinición de los límites de lo público y lo privado, y, aún más importante, sobre la apropiación y mercantilización de los comportamientos de la ciudadanía recogidos en series estadísticas que constituyen un bien común.

\section{Agradecimientos}

El texto se ha realizado en el marco de la investigación Demografía, migraciones y nuevas fronteras estadísticas: Big Data, Registros continuos de población y Registros administrativos, (CSO201785670-R) subvencionado por el Ministerio de Economía y Competitividad de España. 


\section{REFERENCIAS BIBLIOGRÁFICAS}

Aamore, L. 2013. The Politics of Possibility. Risck and Security Beyond Probability. Duke: Duke University Press.

Argüeso Jiménez, A. 2014. “¿Cómo será el censo de población de 2021 en España?” Índice 60: 13-15.

Argüeso Jiménez, A. 2019. "Los censos de población y viviendas de 2021 en España se basarán en registros administrativos". Índice 74: 10-12.

Bechtold, S. 2012. Lessons learned from a mixed-mode census for the future of social statistics. UNECE.

Courgeau, D. y Eva Lelièvre 1997. "Changing Paradigm in Demography”. Population: An English Selection 1997, 9: 1-10. ffhal-02065593f.

Curtis, B. 2002. "Foucault on governmentality and population: The impossible Discovery". Canadian journal of sociology, 27(4): 505-535. https://doi.org/10.2307/3341588.

Desrosières, A. 2004. La política de los grandes números. Barcelona: Melusina.

Dugmore, K.; Furness, P.; Leventhal, B. y C. Moy, 2011. "Beyond the 2011 census in the United Kingdom. With an international perspective". International Journal of Market Research 53(5): 619-650. https://doi.org/10.2501/ IJMR-53-5-619-650.

Holt, T. 2007. "The official statistics Olympic challenge: wider, deeper, quicker, better, cheaper. (With discussions)", The American Statistician 61, 1-1. https://doi.org/10.1198/000313007X168173.

Kitchin, R. 2013. "Big data and human geography: Opportunities, challenges and risks". Dialogues in human geography $3(3)$ : 262-267. https://doi.org/10.1177/2043820613513388.

Kitchin, R., Gleeson, J. y M. Dodge. 2013. "Unfolding mapping practices: a new epistemology for cartogra- phy". Transactions of the Institute of British Geographers 38(3): 480-496. https://doi.org/10.1111/j.14755661.2012.00540.x

Lutz, R. 2008. Ley y orden. Dominación mediante la administración en el siglo XIX. Madrid: Siglo XXI.

Ramiro-Fariñas, D. 2019. "Hacia un nuevo paradigma en la producción estadística: Las infraestructuras de datos y los registros longitudinales de población". Índice 74: 4-5.

Redfern, P. 1989. "Population registers: some administrative and statistical pros and cons". Journal of the Royal Statistical Society. Series A (Statistics in Society): 1-41. https://doi.org/10.2307/2982819.

Skinner, C. 2018. "Issues and challenges in census taking". Annual Review of Statistics and its Application 5, 49-63. https://doi.org/10.1146/annurev-statistics-041715-033713.

Thygesen, L. 2015. "The use of administrative sources for censuses: Merits and challenges". Statistical Journal of the IAOS 31(3): 381-389. https://doi.org/10.3233/ SJI-150909.

UNECE. 2007. Register-based Statistics in the Nordic Countries. Review of the best practiques with focus on population and social statistics. UNECE.

Vikström, P., Edvinsson, S. y A. Brändström, 2002. "Longitudinal databases-sources for analyzing the life-course: Characteristics, difficulties and possibilities". History and Computing 14(1-2), 109-128. https://doi. org/10.3366/hac.2002.14.1-2.109.

Zhang, L. C. 2012. "Topics of statistical theory for register-based statistics and data integration". Statistica Neerlandica, 66(1) 41-63. https://doi.org/10.1111/j.14679574.2011.00508.x.

Zuboff, Sh. 2019. The Age of Surveillance Capitalism. Londres: Profile Books.

ANDREU DOMINGO I VALLS es Doctor en Sociologia, investigador y subdirector del Centre d'Estudis Demogràfics (CED) en la Universitat Autònoma de Barcelona (UAB). Especializado en migraciones internacionales, formación de la família y teoria sobre la población. Investigador Principal del Grup d'Estudis de Demografia i Migracions (GEDEM; http://gedemced.uab. cat/es), reconocido como grupo de investigación consolidado por la Generalitat de Cataluña. Presidente de la Asociación de Demografía Histórica (ADEH).

XIANA BUENO es doctora en Demografía por la UAB y actualmente es investigadora asociada al Centre d'Estudis Demogràfics (CED). Sus líneas de investigación se centran en las migraciones internacionales, desigualdad de género, pautas de formación familiar y políticas familiares. Desde 2006 es miembro del Grup d'Estudis de Demografia i Migracions (GEDEM; http://gedemced.uab.cat/es). Entre 2014 y 2018 hizo su postdoctorado en el Departamento de Sociología de la Universidad de Harvard. En el periodo 2016-2019, obtuvo financiación de las prestigiosas Marie Sklodowska Curie Global Fellowships.

ROCíO TREVIÑO MARURI es Doctora en Sociología por la Universitat Autònoma de Barcelona (UAB) e investigadora del Centre d'Estudis Demogràfics (CED). Está vinculada como investigadora en el Grup d'Estudis de Demografia i Migracions (GEDEM) reconocido como Grupo de Investigación Consolidado por el gobierno de la Generalitat de Cataluña, y especializado en el estudio de las migraciones internacionales Ha participado en numerosos proyectos nacionales e Internacionales relacionados sobre el análisis de las desigualdades de género en el ámbito de la familia y mercado de trabajo. 